\title{
Review: exercise reduces bone loss from the spine in postmenopausal women
}

\author{
Bonaiuti D, Shea B, Iovine R, et al. Exercise for preventing and treating osteoporosis in postmenopausal women. Cochrane \\ Database Syst Rev 2002;(3):CD000333 (latest version 27 Feb 2002).
}

\section{QUESTION: In postmenopausal women, does exercise slow bone loss or have an effect on axial and appendicular bone density?}

\section{Data sources}

Studies were identified by searching Medline, EMBASE/ Excerpta Medica, CINAHL, HealthSTAR, Sports Discus, the Cochrane Controlled Trials Register, PEDro, and the specialised registry of the Cochrane Musculoskeletal Group (all from 1966 to January 2000). Bibliographies of relevant articles were reviewed, and experts in the field were contacted for additional studies and data.

\section{Study selection}

Studies published in any language were selected if they were randomised controlled trials (RCTs) comparing an exercise programme (eg, walking, callisthenics, resisted strengthening) assumed to be adequate to improve aerobic capacity or adequate to improve both aerobic capacity and muscle strength with standard treatment (eg, usual activity or placebo with or without drug consumption), and participants were healthy postmenopausal women $45-70$ years of age.
For correspondence: Ms B Shea, Institute of Population Health University of Ottawa, Ottawa, Ontario

Canada.

beushea@uottawa.ca

\section{Data extraction}

Data were extracted on sample size, details of the intervention, study quality, and outcomes. Main outcomes included percent change (loss) from baseline of bone mineral density (BMD) per year and number of fractures of the hip, vertebrae, and wrist at 1 and 2 years of follow up.

\section{Main results}

18 RCTs ( $\mathrm{n}=1423$ ) met the selection criteria. Metaanalyses were done using a fixed effects model for homogeneous outcomes and a random effects model for outcomes with significant heterogeneity. (1) Aerobic exercise and weight bearing. Bone loss was lower in the exercise group than in the control group for lumbar spine measurements (table). The groups did not differ for hip and wrist measurements (table). (2) Aerobic exercise. Bone loss was lower in the exercise group than in the control group for lumbar spine and wrist measurements (table). The groups did not differ for hip measurements (table). (3) Resistance exercises for specific muscles. Bone loss was lower in the exercise group than in the control group for lumbar spine measurements (table). The groups did not differ for hip and wrist measurements (table). (4) Simple walking. Bone loss was lower in the exercise group than in the control group for hip measurements (weighted mean difference $0.92,95 \%$ CI 0.21 to 1.64 ). The groups did not differ for lumbar spine measurements.

\section{Conclusions}

In postmenopausal women, aerobic, aerobic and weight bearing, and resistance exercises reduce bone loss from the spine. Aerobic exercise also reduces bone loss from the wrist. 


\title{
Review: aerobic exercise improves cardiovascular fitness and tender points in fibromyalgia
}

\author{
Busch A, Schachter CL, Peloso PM, et al. Exercise for treating fibromyalgia syndrome. Cochrane Database Syst Rev \\ 2002;(3):CD003786 (latest version 13 Dec 2001).
}

\section{QUESTION: In patients with fibromyalgia, is exercise training effective for improving symptoms such as physical function, pain, and tender points?}

\section{Data sources}

Studies were identified by searching Medline (19662000), CINAHL (1982-2000), EMBASE/Excerpta Medica (1974-2000), HealthSTAR (1990-2000), Sports Discus (1975-2000), the Cochrane Controlled Trials Register (2000, Issue 4), and reviewing bibliographies of relevant articles.

\section{Study selection}

Studies published in any language were selected if they were randomised controlled trials (RCTs) comparing an intervention that included an exercise component with an untreated control or other non-exercise interventions and patients had a diagnosis of fibromyalgia based on published criteria.

\section{Data extraction}

2 reviewers independently extracted data on sample size, details of the intervention, study quality, and outcomes. Main outcomes included pain, tender points, physical function, global wellbeing or perceived improvement, self efficacy, psychological function, fatigue, and sleep.

\section{Main results}

16 RCTs that evaluated 23 exercise interventions for fibromyalgia met the selection criteria. Types of interventions included aerobic training, flexibility training, strength training, and mixed or composite training. 13 RCTs were of moderate to high methodological quality, and 8 met the American College of Sports Medicine training guidelines. 4 RCTs (also classified as high quality training studies) with similar interventions (cycle ergometry, aerobic dance, whole body aerobics, indoor walking) and similar outcome measures were included in the meta-analysis using a random effects model. The aerobic exercise group showed greater improvement than the control group for cardiovascular fitness (standardised mean difference [SMD] 0.79, 95\% CI 0.37 to 1.21 ) and tender points (SMD 1.19, CI 0.64 to 1.75). The groups did not differ for pain (SMD 0.57, CI -1.20 to 0.06$)$.

\section{Conclusion}

In patients with fibromyalgia, aerobic exercise training is effective for improving cardiovascular fitness and tender points.
Source of funding: not stated.

For correspondence: Professor A Busch, School of Physical Therapy, University of Saskatchewan,

Saskatoon,

Saskatchewan, Canada. angela.busch@usask.ca

\section{COMMENTARY — continued from previous page}

An earlier review found that physical activity reduced overall mortality risk in postmenopausal women and that benefits increased with increased activity. ${ }^{2}$ The review by Bonaiuti $e t$ al focused on healthy postmenopausal women $45-70$ years of age and outcomes of change in bone density and wrist fractures at 1-2 years. However, fractures were reported in only 1 study. This lack of information on the effect of exercise on fracture rates is crucial because it is unclear that changes in bone density lead to changes in fracture risk. ${ }^{3}$ Further research that aims to modify risk of fracture through interventions such as exercise must follow up women to ascertain the effect on fractures. Busch et al found evidence of limited but important changes in symptoms. Aerobic exercise had beneficial effects for patients with fibromyalgia, with improvements in aerobic performance and tender points. Pain, fatigue, and psychological functioning did not improve. The authors did not address sex differences in the effects of exercise programmes on fibromyalgia symptoms because the primary studies had varied sample composition-some included only women, some had mixed samples, and some were unidentified.

The reviews by Bonaiuti $e t$ al and Busch $e t$ al provide nurses working in a range of settings (from acute orthopaedic surgery to primary care, well women clinics) with further evidence of the potential positive effects of physical activity on outcomes in postmenopausal women and patients with fibromyalgia. Exercise clearly has beneficial effects on selected outcomes in both conditions. Clients must be apprised of the specific benefits that can realistically be expected. Nurses must also be aware of the multiple barriers facing people who are thinking of initiating an exercise programme and help clients to explore strategies to overcome such barriers.

Donna Ciliska, RN, PhD Professor, School of Nursing, McMaster University Clinical Consultant, Hamilton Social and Public Health Services Hamilton, Ontario, Canada

1 Report offers new eating and physical activity targets to reduce chronic disease risk. (5 Sep 02). http://www4.nationalacademies.org/news.nsf/isbn/

2 Kushi LH, Fee RM, Folsom AR, et al. Physical activity and mortality in postmenopausal women. JAMA 1997;277:1287-92. Sarkar S, Mitlak BH, Wong M, et al. Relationships between bone mineral density and incident vertebral fracture risk with raloxifene
2002;17:1-10. 